\title{
A STURMIAN THEOREM FOR PARTIAL DIFFERENTIAL EQUATIONS
}

\section{R. M. REDHEFFER ${ }^{1}$}

1. Introduction. In a paper with which the reader is presumed to be familiar [1], Hartman and Wintner have shown how the classical theorems of Sturm concerning the zeros of solutions may be extended to a wide class of partial differential equations. The result to be established here is related to that of [1], though neither includes the other. Whereas [1] compares the equations

$$
L(u)+\phi u=0, \quad L^{*}(u)+\phi^{*} u=0
$$

with different operators $L, L^{*}$ and functions $\phi, \phi^{*}$, the present analysis requires $L=L^{*}$. By way of compensation, however, it will be seen that the assumptions on $L$ and on the region are somewhat weaker than those of [1]. For this reason the methods of Hartman and Wintner are not directly applicable; and in fact, our procedure has little in common with theirs.

2. Definitions and notations. Let a nonlinear operator $L$ be defined by

$$
L(w)=\sum a_{i j}\left(x_{k}, w_{k} / w\right) w_{i j}
$$

where

$$
\begin{aligned}
w & =w\left(x_{1}, x_{2}, \cdots, x_{n}\right), \\
w_{i} & =\partial w / \partial x_{i}, \\
w_{i j} & =\partial^{2} w / \partial x_{i} \partial x_{j}
\end{aligned}
$$

and where $a_{i j}\left(x_{k}, w_{k} / w\right)$ is an abbreviation for

$$
a_{i j}\left(x_{1}, x_{2}, \cdots, x_{n} ; w_{1} / w, w_{2} / w, \cdots, w_{n} / w\right) .
$$

It is supposed that $a_{i j}$ is a well-defined function of its $2 n$ arguments for all values relevant to the ensuing discussion. Similar remarks apply to the functions $\phi$ and $\phi^{*}$ introduced in the sequel; e.g.,

Presented to the Society, November 12, 1955 under the title Characteristic values. zeros, and comparison; received by the editors June 11, 1956.

1 National Science Foundation Fellow.

This paper was prepared in part under the sponsorship of the Office of Naval Research and the Office of Ordnance Research. Reproduction in whole or in part is permitted for any purpose of the United States Government. 


$$
\phi\left(x_{k}, w_{k} / w\right)=\phi\left(x_{1}, x_{2}, \cdots, x_{n} ; w_{1} / w, w_{2} / w, \cdots, w_{n} / w\right) .
$$

To avoid difficulty when $w=0$, and to postulate the required smoothness, we introduce the following definition:

Definition 1. In a region $R$, the function $w$ is an admissible partial solution of

$$
L(w) / w \geq \phi\left(x_{k}, w_{k} / w\right), \quad\left(x_{1}, \cdots, x_{n}\right) \in R
$$

if $w_{i}$ is differentiable in $R$ for each $i$, and if (2) holds at every point of $R$ where $w \neq 0$.

The differentiability ensures $w_{i j}=w_{j i}$ by a known theorem of Young [3], and this in turn yields the usual condition

$$
\left(w_{i j}\right) \leq 0
$$

at a maximum of $w$. (Here the notation (3) means that the matrix $\left(w_{i j}\right)$ is negative semidefinite; and similarly in other cases.)

It is desirable to specify the behavior of $w$ on the boundary of $R$ without, however, requiring continuous boundary values.

Definition 2. If $w$ is defined in a region $R$, we say

$$
\text { inf } \sup w \leq m \text { at the boundary, }
$$

when there is a sequence of compact subsets

such that

$$
R_{n} \subset R_{n+1} \subset R, \quad \lim R_{n}=R
$$

$$
\sup w \leq m+1 / n \text {, }
$$

the sup being taken over the boundary of $R_{n}$.

It is not required that $w \leq m$ in a neighborhood of the boundary; indeed, $\lim \sup w=\infty$ at the boundary is permissible. The notation "inf sup" suggests that one takes the sup on the boundary of $R_{n}$, but the inf with respect to the various choices of $R_{n}$ that are possible.

3. The theorem. These preliminaries enable us to state the following Sturmian theorem, analogous to that of [1]:

ThEOREM. Let $u$ be an admissible partial solution of the boundaryvalue problem

$$
\begin{aligned}
& L(u) / u \geq \phi\left(x_{k}, u_{k} / u\right), \\
& \text { inf } \sup |u| \leq 0 \text { at the boundry } \\
& u \neq \equiv 0
\end{aligned}
$$

in a region $R$, where $L$ is the operator (1). Suppose $\left(a_{i j}\right) \geqq 0$, and let $\phi^{*}$ be a function such that 


$$
\phi^{*}\left(x_{k}, s_{k}\right)<\phi\left(x_{k}, s_{k}\right)
$$

for all relevant values of the $2 n$ arguments. Then every admissible partial solution of

$$
L(v) / v \leq \phi^{*}\left(x_{k}, v_{k} / v\right)
$$

satisfies inf $|v|=0$ in $R$.

If inf $|v|>0$, we may define $w$ by

$$
u=v w .
$$

Since $u \neq 0$, and since inf $\sup |u| \leq 0$ at the boundary, it is easily established that $w$ attains a positive maximum or negative minimum at an interior point of $R$. At this point we have $u v \neq 0$, so that (4) and (5) hold. Also

$$
\begin{aligned}
u_{i} / u & =v_{i} / v, \\
u_{i j} / u & =v_{i j} / v+w_{i j} / w
\end{aligned}
$$

and hence, at the point in question,

$$
\begin{aligned}
\phi\left(x_{i}, v_{i} / v\right) & =\phi\left(x_{i}, u_{i} / u\right) \leq L(u) / u \\
& =\sum a_{i j}\left(x_{k}, u_{k} / u\right) u_{i j} / u \\
& =\sum a_{i j}\left(x_{k}, v_{k} / v\right)\left[v_{i j} / v+w_{i j} / w\right] \\
& =L(v) / v+\sum a_{i j} w_{i j} / w \\
& \leq \phi^{*}\left(x_{i}, v_{i} / v\right)+\sum a_{i j} w_{i j} / w .
\end{aligned}
$$

Since $\left(a_{i j}\right) \geq 0$ everywhere, and since

$$
\left(w_{i j} / w\right) \leq 0
$$

at the extremum, the theorem of Schur and Fejér [2] implies

$$
\sum a_{i j} w_{i j} / w \leq 0 \text {. }
$$

Thus, the condition $\phi>\phi^{*}$ is violated; and this completes the proof.

4. Concerning nonlinearity. In many problems of the sort considered here the nonlinear character is really fictitious; for, an equation such as (2) becomes linear when one substitutes in the values of $w$ and $w_{i}$. If $u$ and $v$ satisfy nonlinear equations of the type contemplated in the theorem then they satisfy linear homogeneous equations.

It is not possible to proceed thus in the proof, for the following reason. When we carry out that process for $u$ and $v$ we get two equations which are both linear, to be sure; but the equations are otherwise quite unrelated. The coefficients for the $v$ equation are evaluated 
by substituting $v$, while those of the $u$ equation are found from $u$. Since there is no reason to expect $u=v$, part of the hypothesis disappears in the process, and the proof cannot be completed. For the same reason, the hypothesis does not ensure $L(v) / v \leq L(u) / u$.

Actually, the theorem is false when the functional form of the coefficients is unrestricted. Taking $n=2, x_{1}=x, x_{2}=y$, let an operator $L$ be defined by

$$
L(u)=u_{x x}\left[1+4\left(\frac{u-\cos 2 x \cos 2 y}{\cos x \cos y+\cos 2 x \cos 2 y}\right)^{2}\right]
$$

in analogy with (1) and let $R$ be the square

$$
|x|<\pi / 4, \quad|y|<\pi / 4 \text {. }
$$

Then $a_{i j}$ is well-defined, $a_{11} \geqq 1, a_{12}=a_{21}=a_{22}=0$, and hence $\left(a_{i j}\right) \geq 0$. The function

$$
u=\cos 2 x \cos 2 y
$$

satisfies

$$
L(u) / u=-4, \quad u=0 \text { at the boundary. }
$$

The function

$$
-v=\cos x \cos y
$$

satisfies

$$
L(v) / v=-5, \quad v \neq 0 \text { in } \bar{R} .
$$

Hence the theorem is not valid for this operator $L$, and the special form of the coefficients must actually play a role in the analysis.

5. Concluding remarks. We wish to indicate how the condition $\phi^{*}<\phi$ may be replaced by $\phi^{*} \leq \phi$, though detailed proofs are not presented here. The operator $L$ is said to be weakly definite if $\left(a_{i j}\right) \geq 0$ and if, for each compact subset $S$ of $R$, there is a constant vector $c_{i}$ such that

$$
\sum a_{i j} c_{i} c_{j}>0 \text { throughout } S .
$$

When $L$ is weakly definite in this sense, and when

$$
a_{i j} \in C^{(1)}, \quad \phi \in C^{(1)}, \quad \phi^{*} \in C^{(1)}, \quad u \in C^{(2)}
$$

it is not difficult to show that $\phi \leq \phi^{*}$ is sufficient to yield the conclusion of the theorem. In fact, the inequality

$$
v L(u)-u L(v) \geq \phi\left(x_{k}, u_{k} / u, v_{k} / v\right)
$$


with mild restrictions on $\phi$ ensures that $u / v$ satisfies the maximum principle; and this is the property which underlies the present analysis.

\title{
BiBLIOGRAPHY
}

1. P. Hartman and A. Wintner, On a comparison theorem for self-adjoint partial differential equations, Proc. Amer. Math. Soc. vol. 6 (1955).

2. H. Bateman, Partial differential equations of mathematical physics, New York, Dover, 1944, pp. 37, 135-138.

3. De la Vallee Poussin, Cours d'analyse, vol. I, New York, Dover, 1956, p. 118. University of California, Los Angeles

\section{A NOTE ON LINEAR ORDINARY DIFFERENTIAL EQUATIONS}

\author{
STEPHEN P. DILIBERTO ${ }^{1}$
}

Let

$$
\frac{d x}{d t}=A(t) x,
$$

where $x$ is an $n$-column vector

$$
\left(\begin{array}{l}
x_{1} \\
: \\
x_{n}
\end{array}\right)
$$

and $A=\left(a_{i j}(t)\right)$ where $a_{i j}(t)$ are continuous real valued functions of time $(-\infty<t<+\infty)$. Let $y^{1}(t), \cdots, y^{n}(t)$ be any $n$-linearly independent solutions of (1) defined for all $t$. Let $B^{1}(t), \cdots, B^{n}(t)$ be the $n$ normal-orthogonal vectors obtained from the set $\left\{y^{i}\right\}$ by the GramSchmidt orthogonalization process. Let $B(t)$ be the orthogonal matrix whose $j$ th column is $B^{j}(t)$, and introduce a new variable $u$ (an $n$ column vector) defined by

$$
x=B(t) u .
$$

$u$ satisfies the linear differential equation

Received by the editors August 23, 1956.

1 The author is indebted to the Office of Scientific Research for a research grant 1954-1955, during which time this work was undertaken. 